\title{
Boosting Seniors' Confidence by Enhancing User Instructions
}

\author{
Nicole Loorbach \\ University of Twente \\ n.r.loorbach@utwente.nl
}

\author{
Joyce Karreman \\ University of Twente \\ j.karreman@utwente.nl
}

\author{
Michaël Steehouder \\ University of Twente \\ m.f.steehouder@utwente.nl
}

\begin{abstract}
This paper describes a study on enhancing user instructions to boost seniors' (60 - 70 yrs) confidence in using instructional texts. We developed one basic and two enhanced versions of user instructions for a cellular telephone. The first enhanced version contained control steps - where possible - so users can check if procedures were performed correctly. The second enhanced version contained personal stories (both anecdotes and testimonials) that describe how a 68-year-old woman struggled a bit with the instructions, but always succeeded in reaching her goal. We used the plus-minus method to measure seniors' reactions to control steps and personal stories in user instructions. The results show that although control steps do not particularly stand out in enhanced user instructions, a vast majority of seniors (95\%) do value them positively. Personal stories stood out more than control steps, but were valued positively by fewer seniors. Still, a majority of 65 percent suggested including such stories in user instructions. As a next step, we will conduct an experimental study to test for the individual effects of control steps and personal stories on seniors' confidence, on their motivation, and on the usability of the user instructions.

Keywords: confidence, motivation, usability, seniors, user instructions.
\end{abstract}

\section{Introduction}

For a long time, user instructions were considered as purely instrumental documents: Instructions had to enable readers to perform tasks with an accompanying device. And even though this still remains the main purpose of user instructions, views on how to accomplish this have changed over the years. The traditional view seemed to assume that when the instructions were correct, readers would automatically be able to use the accompanying device well. In other words, instrumental discourse alone should be enough for readers to reach their goals with a device. Or as Moore [1, p.166] stated: "Instrumental discourse does not persuade like rhetoric; it shows a user how to perform an action. ... Rhetorical communications and salespeople may persuade customers to buy specific hardware and software, but after the sale, the customers require no persuading to read and apply the installation and operating instructions. External circumstances obligate them to perform those tasks so they can use their new purchases." Even though this line of thought sounds logical, many users of instrumental discourse have experienced otherwise and ended up not being able to use their new purchases as well as they would like to.

Research has shown that contrary to common beliefs, consumers generally do use instruction manuals. But when they do, they usually only scan the user instructions or use it as reference $[2,3]$. Users might not need any persuasion to start reading the instructions of a device they just bought, but the fact remains that the process of using instructions and getting to know a new purchase is usually not a flawless one. People will encounter problems and then what? Truth is, many users will abandon reading the instructions when they feel what they're looking for is not provided in the manual. Then it becomes easier to just give up and ask a friend or relative to help out or even worse, to do it for them. We hold the opinion that if user instructions were used more thoroughly, then users would experience fewer problems, use their devices to a fuller extent and have a smaller chance of damaging a device as a result of incorrect use. On top of that, manufacturers would probably benefit from a decrease in helpdesk calls.

So even though users of devices don't seem to need persuasion to start reading user instructions, enhancing user instructions to motivate users to continue reading does seem called for. Current views on the design of technical documents still assume that above all, instructions should enable readers to perform tasks with the accompanying device. But in order to accomplish this, the instructions should motivate readers to keep on reading once they have started doing so.

Goodwin [4, p.100] argues that "Audience motivation ... plays a vital role in effective technical communication. Specifically, a good technical writer must keep a reader reading long enough and carefully enough to become competent at specific tasks". Horton [5] advocates a similar view, suggesting that technical documents should motivate readers, and that technical writers should take responsibility for making the reader notice, understand, and act on the information. Horton labels instructional documents according to the traditional view on technical 
documents as "friendly documents", whereas documents that are enhanced by motivational elements can be seen as "seductive documents". He claims that friendly documents enable readers to do and to find: They present information clearly, make a case, and are readable. Seductive documents, however, impel readers to do: They show, teach, convince, and get read. So, according to Horton, friendly documents allow access to information, if a reader is motivated and tries to find it. Seductive documents go further by supplying the motivation.

Previous research on supplying motivation in user instructions indicates that enhancing user instructions by focusing on users' confidence is most promising [9]. Boosting user' confidence not only improves usability aspects of the instructional text, but it also increases user's motivation to continue using the instructions.

\section{Previous research on supplying motivation in user instructions}

So far, we have conducted two studies to investigate the effects of supplying motivation in user instructions for telephones [see 6 for an overview of these studies]. To supply motivation in user instructions, we added so-called motivational elements, which we defined as textual additions or modifications to user instructions with the aim of motivating the reader, without changing the content of the actual instructions.

Our first study was exploratory in nature and tested for collective effects of six types of motivational elements in user instructions for a fixed, wireless telephone [see 7 for an elaborate description]. Forty students performed twelve tasks and filled out questionnaires. The motivational elements in this study positively affected one of ISO's three usability aspects [8], namely user satisfaction with the instruction manual. A ceiling effect occurred for the other two usability aspects, namely effectiveness and efficiency of task performance. This was most probably due to the students' relatively high level of prior knowledge about and experience with telephone menu structures.

Our second study [see 9 for an elaborate description] not only tested for effects of motivational elements on usability, but also on actual user motivation to keep using the instructions once they had started doing so. Also, we decided to ask 79 seniors (60-70 yrs) instead of students to perform tasks and fill out questionnaires, in an attempt to avoid ceiling effects when it comes to users' effectiveness and efficiency in performing tasks. Seniors belong to a user group that is known for being less experienced with relatively new technology devices like cellular telephones [10]. This time, we designed one basic version and three motivational versions of user instructions for a cellular telephone. The motivational versions were designed on the basis of the ARCS Model of Motivational Design [11 - 13] and focused on respectively
1) gaining and maintaining user' attention, 2) increasing users' sense of relevance of the instructions, and 3) increasing users' confidence in using the instructions. The results of this study showed positive effects of motivational elements that focus on relevance and confidence. These elements positively affected seniors' effectiveness in performing tasks. Also, confidence elements positively affected seniors' persistence in trying to complete tasks. In other words: seniors who used the enhanced version that focused on increasing confidence not only performed more tasks correctly, but also gave up using the instructions less easily than seniors using the basic version.

The results of these previous studies on supplying motivation in user instructions for telephones led us to believe that enhancing instructions by focusing on users' confidence will be most beneficial for users.

\section{Boosting confidence to increase motivation and usability}

In creating strategies to boost seniors' confidence in using instructions, we rely on Bandura's social cognition theory [14]. In this theory, perceived self-efficacy is defined as "people's judgments of their capabilities to organize and execute courses of action required to attain designated types of performances" (p. 391). In our case, perceived self-efficacy is made up by judgments of seniors of their capabilities to organize and execute courses of action required to perform assigned tasks with a provided cellular telephone and the accompanying user instructions. We will use seniors' judgments of selfefficacy as a measure of their confidence.

Bandura states that "judgments of efficacy also determine how much effort people will expend and how long they will persist in the face of obstacles or aversive experiences. The stronger their perceived self-efficacy, the more vigorous and persistent are their efforts. When beset with difficulties, people who are plagued by selfdoubts about their capabilities slacken their efforts or give up altogether, whereas those who have a strong sense of efficacy exert greater effort to master the challenge." [14, p. 394]

According to Keller [11, p. 391], "effort refers to whether the individual is engaged in actions aimed at accomplishing the task. Thus, effort is a direct indicator of motivation." So by increasing users' confidence in using instructions, their motivation to use the instructions should increase as well. According to the previously described current view on the design of technical documents, this is exactly what is needed to enable users to perform tasks with the device in question.

All in all, we expect that boosting users' confidence by enhancing user instructions will cause senior users to be 
more motivated to keep on reading once they have started doing so, which in turn will lead to an increased usability of the user instructions, measured as task effectiveness, task efficiency, and satisfaction. We are setting up a study to test these hypotheses about the individual effects of two specific confidence enhancements for user instructions: providing 1) control steps, and 2) personal stories. This paper describes the reactions of 20 seniors to these enhancements in user instructions.

\section{Providing control steps}

Bandura [15] describes four principal sources of selfefficacy beliefs, of which "enactive mastery experiences that serve as indicators of capability" (p. 79) is the first. He explains that "enactive mastery experiences are the most influential source of efficacy information because they provide the most authentic evidence of whether one can muster whatever it takes to succeed. Successes build a robust belief in one's personal efficacy." Keller [11] also acknowledges the importance of successes in building efficacy, or in his words expectancy. In his explanation of his first strategy to increase expectancy ("Increase expectancy for success by increasing experience with success", p.418), he states: "If a person has a generally low expectancy for success or a specific history of failure in a given area, then a series of meaningful successes in that area will improve the person's expectancy for success" (p. 419).

By providing control steps - where possible - at the end of procedures in user instructions, we hope to stimulate readers to actually experience successes, because these steps enable users to check if procedures were performed correctly. Control steps should first of all take away possible doubts about whether or not having performed a procedure correctly. If success was the case, control steps are a confirmation of indeed having succeeded. In the opposite situation, control steps will inform users that a procedure was not performed correctly and as such allow for a retry and indirectly for success after all.

In our second study [see 9], providing control steps was one of the four strategies aimed at increasing confidence. These strategies had collective positive effects on the effectiveness of task performance and on senior users' persistence in trying to accomplish tasks. Now, we are interested in individual effects of providing control steps. See Figure 1 for an example of control steps in user instructions.

\section{Providing personal stories}

Bandura describes the second source of self-efficacy beliefs as "vicarious experiences that alter efficacy beliefs through transmission of competencies and comparison with the attainments of others" [15, p. 79]. This source is better known as modeling. The personal stories should stimulate users to feel that they too can perform certain tasks with the telephone. According to Bandura, "seeing or visualizing people similar to oneself perform successfully typically raises efficacy beliefs in observers that they themselves possess the capabilities to master comparable activities. They persuade themselves that if others can do it, they too have the capabilities to raise their performance" (p. 87).

The personal stories in the enhanced version of the user instructions are either anecdotes or testimonials that describe how a 68-year-old woman named Ria Damhuis struggled a bit with the instructions, but always succeeded in reaching her goal. Each story is accompanied by a picture of Ria to stimulate the process of modeling.

In both our previous studies [7, 9], we used personal stories to design motivational versions of the user instructions. In our first study, there was only one motivational version which comprised six types of motivational elements, of which personal stories were one. In our second study, we used personal stories as one of four strategies in designing the relevance version of the instructions. These four strategies had collective positive effects on the effectiveness of task performance. Now, we are interested in individual effects of providing personal stories. We will use personal stories to boost users' confidence in using the instructions.

So why are we currently using personal stories to boost confidence when we used them to increase relevance in our previous study? In the light of both the ARCS Model of Motivational Design and the aim of our current study, this is justified. Visser [16, p. 145] acknowledges that "the four dimensions of the ARCS model are more to be seen as a four-dimensional look at motivation and thus cannot be strictly separated; often indeed the categories go smoothly over from one dimension into the other". In the process of narrowing down the amount of strategies to motivate users, we don't necessarily seek out strategies that only influence users' confidence in using the instructions: We are looking for strategies that work well when it comes to boosting users' confidence. From that perspective, automatically focusing on relevance as well is a welcome addition as opposed to a nuisance. The nature of personal stories automatically calls for some focus on relevance: When a user describes his or her actions with a cellular telephone, the relevance of these actions to the user almost inevitably shines through. In our previous studies, personal stories especially focused on relevance. Now, the focus will lie on boosting confidence. See Figure 1 for an example of personal stories in user instructions. 
Figure 1. Example pages (in Dutch).

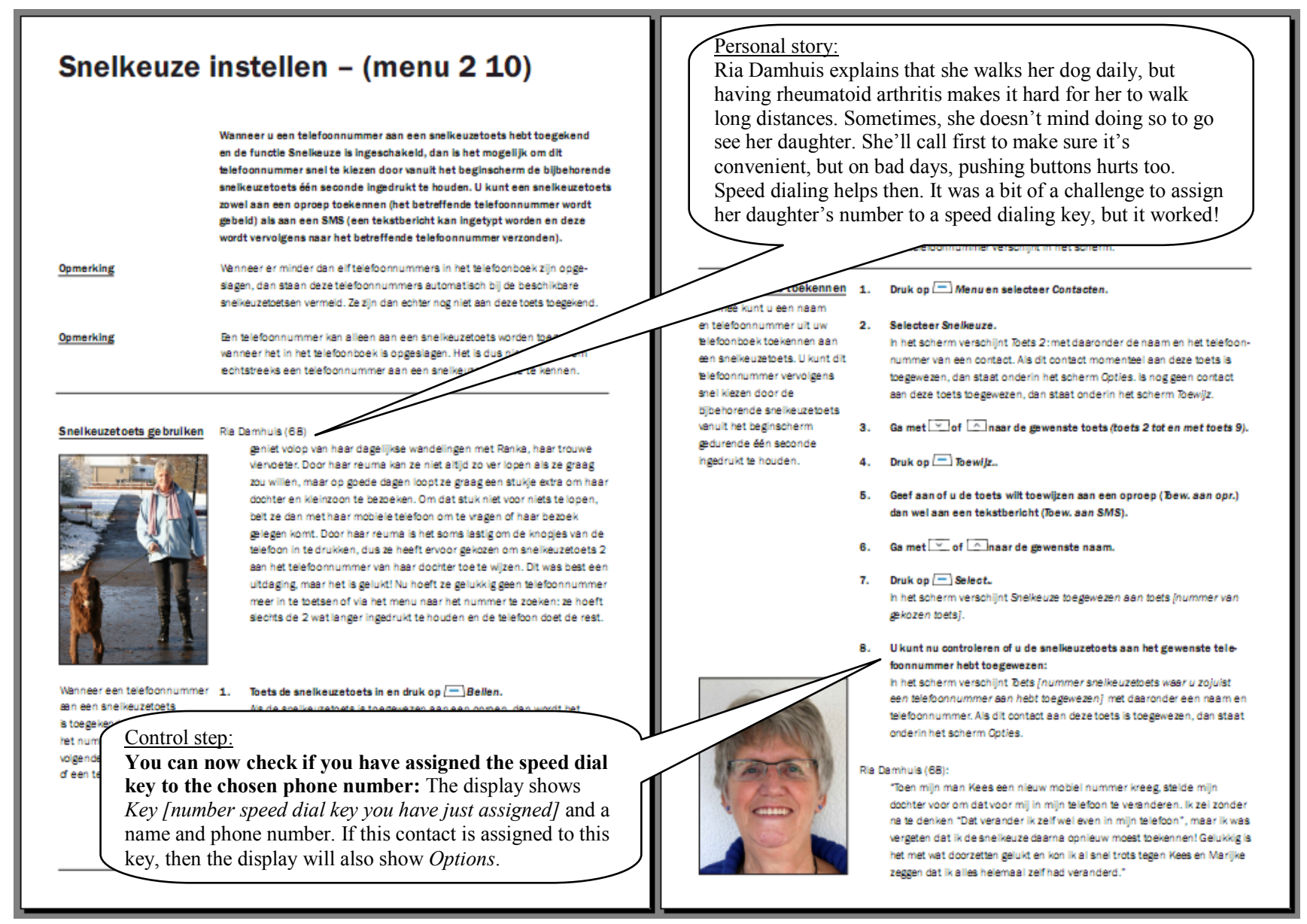

\section{Confidence-boosting user instructions: what seniors think}

Before testing for individual effects of control steps and personal stories on confidence, motivation, and usability, we wanted to know how seniors respond to these enhancements for user instructions. We visited with twenty seniors (6 males, 14 females) between 60 and 70 years of age. Besides pre-testing some questionnaires on aspects of confidence, we used the plus-minus method to invoke seniors' responses to a section of user instructions for a cellular telephone. This section on speed dialing consisted of four pages and contained both control steps and personal stories.

The plus-minus method involves asking participants to read a text from start to finish and to mark their positive and negative reading experiences with pluses and minuses, respectively, in the margin. Pluses and minuses may be assigned to all sorts of text elements (from chapters to words) and for various reasons (e.g., comprehensibility, appreciation, relevance of the information). After that, individual interviews are held, focusing on the reasons for every plus and minus $[17, p$. 123].

When participants placed a plus or minus next to a control step or personal story and their explanation of this plus or minus indicated that the plus or minus was placed as a response to the strategy in question (and not, for example, to a particular word in the enhancement), then we recorded this entry as a "spontaneous" plus or minus. When participants had not put any plus or minus next to a control step or personal story, then the interviewer asked for each enhancement: "If you had to place a plus or minus, what would it be? When it is up to you, would you value this in user instructions or would you leave this out?" The resulting pluses and minuses were recorded as "forced" pluses and minuses. 


\section{Seniors' responses to control steps}

As stated earlier, there were four possible responses to control steps in the speed dialing section of the user instructions (see Table 1).

Table 1. Seniors' responses to control steps.

\begin{tabular}{|l|c|c|}
\hline \multicolumn{1}{|c|}{ Response } & Frequency & Percent \\
\hline Spontaneous plus & 2 & 10 \\
Spontaneous minus & 0 & 0 \\
Forced plus & 17 & 85 \\
Forced minus & 1 & 5 \\
\hline
\end{tabular}

It is interesting to see that only ten percent of the responses were spontaneously given. This suggests that control steps do not particularly stand out in enhanced user instructions. On top of that, not a single spontaneous minus was given to control steps, indicating that providing this enhancement probably will not offend or annoy senior readers.

Nineteen out of 20 participants responded positively to the control steps: if seniors had to choose between including control steps in user instructions or omitting them, 95 percent of them would include them.

The interviews on the reasons for the pluses and minuses showed a similar trend: seniors have positive attitudes towards providing control steps in user instructions. The one person who placed a minus explained: "When I'm calling someone, I don't need the instructions to tell me how to check if I'm actually calling. I can hear whether or not I'm calling". Following are some examples of seniors' explanations for pluses:

- "If you don't perform this procedure very often, then you'll have doubts and this way, you can check whether or not you did it correctly".

- "This allows you to check. That's very important to me. It'll slow things down a bit, but you'll be sure that things went well. ... To me, being in control is important".

- "When you can check that you did something, then you're convinced... Look, it makes you feel secure. ... When I can check and when something has gone right, then I'm actually a little proud of myself. Then I made it happen!"

The explanations convey the impression that control steps could indeed let users experience their successes: If users can check, then they will know when they have done things right and feel secured and hopefully more confident as a result.

\section{Seniors' responses to personal stories}

As with the control steps, there were four possible responses to personal stories in the speed dialing section of the user instructions (see Table 2).

Table 2. Seniors' responses to personal stories.

\begin{tabular}{|l|c|c|}
\hline \multicolumn{1}{|c|}{ Response } & Frequency & Percent \\
\hline Spontaneous plus & 7 & 35 \\
Spontaneous minus & 2 & 10 \\
Forced plus & 6 & 30 \\
Forced minus & 5 & 25 \\
\hline
\end{tabular}

The first thing that stands out is that compared to the responses to control steps, the responses to personal stories are distributed more evenly over the response categories. Forty-five percent of the responses were spontaneous, so personal stories seem to stand out more than control steps. This is not surprising, since each story is accompanied by a picture, and anecdotes and testimonial are not very common in user instructions.

Even though personal stories are usually not included in user instructions, the majority of seniors do value them in instructions: If seniors had to choose between including personal stories in user instructions or omitting them, 65 percent would include them. Following are some examples of seniors' explanations for pluses:

- "These stories make it easy for us. Because we read this story about this lady who walks her dog and wants to visit someone, right? And then I think 'yes, that lady in a sense has the same problem we do'. Here it says 'she managed to get it done, but it cost some effort' and she was proud of achieving that, right? Towards her children. ... A short story like that makes me feel secure".

- "I gave a plus, because [stories] visualize, they speak out... But only in user instructions for seniors. Not for eighteen-year-olds. They don't want that".

- "The fact that she managed to do this is very positive. I would try it too. I would really try it and say to myself 'well, I'm just going for it'.... Stories like this indicate that many people are dealing with this. And that many people sometimes encounter problems doing so. And I just think that's great. Really!"

These are some examples of seniors' explanations for minuses:

"These stories, people won't read them anyway. At least, I wouldn't include them. It's an instruction manual and you want to get as fast and as good as possible to... It is friendlier towards people, but still. 
It allows you to imagine. But then I think 'Does this belong in an instruction manual?' I don't think so."

. "I think it's absurd. Why should I know what this woman did? I don't think this is necessary at all. Just say plainly, well, clearly what needs to be said."

Participants pretty much agreed on their reasons for pluses and minuses. Pluses were most often placed because seniors recognize themselves in the described situation. The major reason to place a minus seems to be that personal stories are regarded as unnecessary in user instructions. Even when seniors regard personal stories as unnecessary, they might still have positive effects on their confidence and motivation, and on the usability of the instructions. As a next step, we will conduct an experimental study in which we will use one basic and two enhanced versions of user instructions for a cellular telephone to test for these effects.

\section{Confidence-boosting user instructions: what seniors do}

We will conduct an experimental study to test for the individual effects of control steps and personal stories on senior users' confidence, their motivation, and on the usability of user instructions for a cellular telephone.

We will use one basic version and two enhanced versions of user instructions for a cellular telephone. We improved the layout of the basic version of our second study [see 9] to design this study's basic version. The first enhanced version contains control steps - where possible so users can check if procedures were performed correctly. The second enhanced version contains 23 personal stories (11 anecdotes and 12 testimonials) throughout the user instructions, all of which describe how a 68-year-old woman named Ria Damhuis struggled a bit with the instructions, but always succeeded in reaching her goal. Each personal story is accompanied by a picture of Ria.

We will visit with 60 seniors at their homes, all of whom are between 60 and 70 years old. We will use randomized stratification to assign participants to one of the three experimental conditions.

To test for differences regarding confidence, we will use the New General Self-Efficacy Scale [18] and we will develop our own Cell Phone Self-Efficacy Scale, according to Bandura's guide for creating self-efficacy scales [19]. We will also use an adapted version of the constructs labeled Comfort, Efficacy and Interest of Jay and Willis' Attitudes Toward Computers Questionnaire [20].

We will measure motivation by self-report measures, using a downsized, 12-item version of the Instructional Materials Motivation Survey [21], and by measuring participants' persistence in trying to accomplish tasks, resulting in one of three scores: 1) complete, 2) give up and 3) persist (stopped by the researcher after 15 minutes of trying).

Usability will be measured by the effectiveness of task performance (number of tasks completed successfully out of three tasks), the efficiency of task performance (time needed to successfully complete tasks) and self-reported satisfaction with the user instructions.

As stated earlier, we expect both confidence-boosting enhancements to user instructions to positively influence senior users' confidence and motivation. In turn, we expect an increased usability of the user instructions in the experimental conditions. On the basis of Bandura's description of the sources of self-efficacy and the link between control steps and the first and most important source on the one hand, and personal stories and the second source on the other, control steps are expected to be most influential in this respect.

\section{References}

[1] Moore, P., Rhetorical vs. instrumental approaches to teaching technical communication, Technical communication, 41:163-173, 1997.

[2] Schriver, K.A., Dynamics in document design: Creating text for readers, John Wiley \& Sons, New York, 1997.

[3] Jansen, C., and S. Balijon. Hoe worden handleidingen gebruikt en gewaardeerd? [How are manuals used and appreciated?] Tijdschrift voor Taalbeheersing, 23(2):132144, 2001.

[4] Goodwin, D., Emplotting the reader: Motivation and technical documentation, Journal of Technical Writing and Communication, 21:99-115, 1991.

[5] Horton, W., Secrets of user-seductive documents: Wooing and winning the reluctant reader, Society for Technical Communication, Arlington VA, 1997.

[6] Loorbach, N., J. Karreman, and M. Steehouder. The Effects of Adding Motivational Elements to User Instructions. IEEE International Professional Communication Conference, Seattle WA, 1-5, 2007.

[7] Loorbach, N., M. Steehouder, and E. Taal. The Effects of Motivational Elements in User Instructions. Journal of Business and Technical Communication, 20(2):177-199, 2006.

[8] International Organization for Standardization. International Standard ISO 9241-11: Ergonomic requirements for office work with visual display terminals 
(VDT's) - Part 11: Guidance on usability, ISO, Geneva Switzerland, 1998.

[9] Loorbach, N., J. Karreman, and M. Steehouder. Adding Motivational Elements to an Instruction Manual for Seniors: Effects on Usability and Motivation. Technical Communication, 54(3):343-358, 2007.

[10] Schwender, C., and C. Köhler. Introducing Seniors to New Media Technology: New Ways of Thinking for a New Target Group. Technical Communication, 53(4):464-470, 2006.

[11] Keller, J.M., Motivational Design of Instruction. In C.M. Reigeluth (Ed.), Instructional-design theories and models: An overview of their current status, 386-434, Lawrence Erlbaum Associates, Hillsdale NJ, 1983.

[12] Keller, J.M., Strategies for Stimulating the Motivation to Learn. Performance \& Instruction, 26(8):17, 1987.

[13] Keller, J.M., Using the arcs motivational process in computer-based instruction and distance education. New Directions for Teaching and Learning, 78:39-47, 1999.

[14] Bandura, A., Social foundations of thought and action: A social cognitive theory, Prentice Hall, Englewood Cliffs NJ, 1986.

[15] Bandura, A., Self-Efficacy: The Exercise of Control, W.H. Freeman and Company, New York, 1997.

[16] Visser, L., The Development of Motivational Communication in Distance Education Support, PrintPartners Ipskamp, Enschede (The Netherlands), 1998.

[17] De Jong, M., and P.J. Schellens. Formative Evaluation in Document Design: Validity of the PlusMinus Method For the Pretesting of Public Information Brochures. Proceedings of the 1998 International Professional Communication Conference, Quebec City (Quebec), 123-130, 1998.
[18] Chen, G., S.M. Gully, and D. Eden. Validation of a New General Self-Efficacy Scale. Organizational Research Methods, 4(1):62-83, 2001.

[19] Bandura, A., Guide for Creating Self-Efficacy Scales. In F. Pajares, and T. Urdan (Eds.), Self-Efficacy Beliefs of Adolescents, 307-337, Information Age Publishing, Greenwich CT, 2006.

[20] Jay, G.M., and S.L. Willis. Influence of Direct Computer Experience on Older Adults' Attitudes Toward Computers. Journal of Gerontology, 47(4):250-257, 1992.

[21] Keller, J.M., Instructional materials motivation survey. Unpublished materials, Florida State University, Tallahassee FL, 1993.

\section{About the Authors}

Nicole Loorbach is a $\mathrm{PhD}$ candidate in the Technical and Professional Communication Department at the University of Twente, the Netherlands. Her research interests include document design and technical communication, in particular motivational elements in technical documents.

Joyce Karreman is an assistant professor in the Technical and Professional Communication Department at the University of Twente. She teaches courses in document design, user support, and academic writing. Her research interests include the use and effects of different information types in instructive texts, and the design and evaluation of texts on the Web.

Michaël Steehouder is the chair of the Technical and Professional Communication Department at the University of Twente. His research interests include document design and rhetoric. He has published textbooks and articles about communication skills, government forms, software manuals, and user instructions. He is past president of STIC, the Dutch society for technical communication, and associate editor of IEEE Transactions on Professional Communication. 